\title{
LETTER
}

\section{Comparison of RIFLE with and without urine output criteria for acute kidney injury in critically ill patients: a task still not concluded!}

\author{
José António Lopes* and Sofia Jorge \\ See related research by Wlodzimirow et al., http://ccforum.com/content/16/5/R200
}

In a recent issue of Critical Care, we read with interest the article by Wlodzimirow and colleagues [1], who prospectively studied the Risk Injury Failure Loss Endstage renal disease (RIFLE) [2] classification with serum creatinine ( $\mathrm{SCr}$ ) and urine output (UO) $\left(\mathrm{RIFLE}_{\mathrm{SCr}+\mathrm{UO}}\right)$ and without UO criteria $\left(\operatorname{RIFLE}_{\mathrm{SCr}}\right)$ for acute kidney injury (AKI) in 260 critically ill patients. RIFLE $_{\mathrm{SCr}}$ significantly underestimated the presence of AKI on admission and during the first week in the intensive care unit and significantly delayed AKI diagnosis. Those are important findings that corroborate the utility of simultaneously using both criteria as proposed by the Acute Dialysis Quality Initiative workgroup [2]. The authors also found that RIFLE $_{\mathrm{SCr}}$ was associated with higher mortality than RIFLE $_{\mathrm{SCr}+\mathrm{UO}}$. This observation should be interpreted with extreme caution, as this association has not been tested by multivariate analysis. Data regarding the impact on mortality of RIFLE defined by SCr and UO or by SCr are not conclusive. For example, in a systematic review, the relative risk for death among studies that used RIFLE $_{\mathrm{SCr}+\mathrm{UO}}$ was lower than in those using RIFLE $_{\mathrm{SCr}}$ [3]. Previously, however, we did not find any difference in terms of mortality for RIFLE ${ }_{\mathrm{SCr}+\mathrm{UO}}$ (Risk, odds ratio (OR) 2.69; Injury, OR 2.01; Failure, OR 3.59; AKI of any category, 2.78; area under the receiver operator characteristic (AUROC), 0.733) or for RIFLE $_{\mathrm{SCr}}$ (Risk, OR 2.63; Injury, OR 2.12; Failure, OR 3.2; AKI of any category, 2.68; AUROC, 0.729) [4]. Therefore, prospective studies with a large number of patients are still needed to better determine the impact on mortality of RIFLE defined by $\mathrm{SCr}+\mathrm{UO}$ criteria or by $\mathrm{SCr}$ criteria.

\section{Authors' response}

Ameen Abu-Hanna, Kama A Wlodzimirow, Marcus Schultz and Catherine SC Bouman

We agree with Lopes and Jorge that multivariate analysis should be attempted when testing whether RIFLE $_{\mathrm{SCr}}$ is associated with higher mortality than $\operatorname{RIFLE}_{\mathrm{SCr}+\mathrm{UO}}$. Essentially the question is whether the group (hereafter $G_{1}$ ) of patients with AKI based on the RIFLE $_{\mathrm{SCr}}$ criteria (regardless of UO) is at higher risk of death than the group (hereafter $G_{2}$ ) classified as having AKI based on the UO criteria only. Additional analysis, not reported in [1], shows that out of admission type, age, gender, weight, Acute Physiology and Chronic Health Evaluation (APACHE) score, Simplified Acute Physiology Score

*Correspondence: jalopes93@hotmail.com

Department of Nephrology and Renal Transplantation, Hospital de Santa Maria, Centro Hospitalar de Lisboa Norte, EPE, Av. Prof. Egas Moniz, 1649-035 Lisboa, Portugal
(SAPS), cardiopulmonary resuscitation, and length of stay, only SAPS was a confounder. Before adjustment for SAPS, patients in $G_{1}$ had 1.64 times the odds of dying than those in $G_{2}$. After adjustment for SAPS, the OR was reduced to $1.45(P=0.0004)$, still confirming our findings, which are in agreement with those of the other study [3].

The seeming contradiction between our findings and those of Lopes and colleagues [4] is easily explained by the significant differences in case mix. In our study, 48.6\% of the RIFLE ${ }_{\mathrm{SCr}+\mathrm{UO}}$ AKI patients were classified as having AKI on the basis of the UO criteria only [1] versus $5.6 \%$ in the study by Lopes and colleagues [4]. Differences in case mix may be attributable to the different inclusion criteria, the Modification of Diet in Renal Disease (MDRD)-based estimation of baseline $\mathrm{SCr}$ in all patients in the previous study [4], which may overestimate AKI based on SCr [5], and the outcome definition. All of these are important factors to consider when comparing studies. 


\section{Abbreviations}

AKl, acute kidney injury; AUROC, area under the receiver operator characteristic; OR, odds ratio; RIFLE, Risk Injury Failure Loss End-stage renal disease; $R_{1 F L E_{S C^{\prime}}}$ RIFLE criteria based on the serum creatinine criteria only; RIFLE $_{\mathrm{SC}+\mathrm{O}^{\prime}}$ RIFLE criteria based on serum creatinine and urine output criteria; SAPS, Simplified Acute Physiology Score; SCr, serum creatinine; UO, urine output.

\section{Competing interests}

The authors declare that they have no competing interests.

\section{Authors' contributions}

JAL and SJ drafted the letter, revised it critically for important intellectual content, and gave final approval of the version to be published.

Published: 23 January 2013

\section{References}

1. Wlodzimirow KA, Abu-Hanna A, Slabbekoorn M, Chamuleau RA, Schultz MJ, Bouman CS: A comparison of RIFLE with and without urine output criteria for acute kidney injury in critically ills. Crit Care 2012, 16:R200.

2. Bellomo R, Ronco C, Kellum JA, Mehta RL, Palevsky P and the ADQI workgroup: Acute renal failure - definition, outcome measures, animal models, fluid therapy and information technology needs: the Second International Consensus Conference of the Acute Dialysis Quality Initiative (ADQI) Group. Crit Care 2004, 8:R204.

3. Ricci Z, Cruz D, Ronco C: The RIFLE criteria and mortality in acute kidney injury: a systematic review. Kidney Int 2008, 73:538-546.

4. Lopes JA, Fernandes P, Jorge S, Gonçalves S, Alvarez A, Costa e Silva Z, França C, Martins Prata M: Acute kidney injury in intensive care unit patients: a comparison between the RIFLE and the Acute Kidney Injury Network classifications. Crit Care 2008, 12:R110.

5. Bagshaw SM, Uchino S, Cruz D, Bellomo R, Morimatsu H, Morgera S, Schetz M, Tan I, Bouman C, Macedo E, Gibney N, Tolwani A, Oudemans-van Straaten HM, Ronco C, Kellum JA: A comparison of observed versus estimated baseline creatinine for determination of RIFLE class in patients with acute kidney injury. Nephrol Dial Transplant 2009, 24:2739-2744.

\section{doi:10.1186/cc11911}

Cite this article as: Lopes JA, Jorge S: Comparison of RIFLE with and without urine output criteria for acute kidney injury in critically ill patients: a task still not concluded! Critical Care 2013, 17:408. 